\title{
A Compact Circularly-Polarized Lens-Horn Antenna with Very Low Axial Ratio and SLL
}

\author{
Fernando Rodríguez Varela , José Luis Besada Sanmartín , Belén Galocha Iragüen
}

\begin{abstract}
Antennas used in radio astronomy and remote sensing applications often require the use of high gain horns whose size can be impractical at lower frequencies. To overcome this problem, lens corrected horns are employed. In this paper, the design process of a lens horn antenna with very low axial ratio and side lobe level is reviewed. An analysis of the lens effect over the radiation pattern and matching is performed and some guidelines on the design process are outlined. Measurements of return loss, isolation, radiation pattern and axial ratio are given showing good agreement with the simulations.
\end{abstract}

Keywords - Potter horn, dielectric lens, phase correction, septum polarizer, side lobe level (SLL), circular polarization.

\section{INTRODUCTION}

High gain horn antennas are widely employed as feeders of large Cassegrain radioastronomy and high capability satellite communications reflectors, as well as independent antennas in applications such as remote sensing calibration [1]. For Cassegrain antennas correct operation, these horns have to radiate narrow beams with low side lobe level (SLL) to illuminate the sub reflector with high beam efficiency, as this reduces the spill-over and antenna noise temperature. Remote sensing calibration consists in the relay of pulses transmitted from a satellite radar by an Earth transponder unit. In this application horns with low SLL are required as well, in order to mitigate multipath signals that can degrade the stability of the calibration process. Both applications usually operate in circular polarization to eliminate Faraday rotation effects. Particularly, in the case of remote sensing calibration extremely low axial ratios are required.

These narrow beams are obtained with electrically large horns (length and aperture), which can pose a problem at L, S and $\mathrm{C}$ band, where the physical dimensions of the horn may result impractical. Aperture size cannot be varied as it is directly related to the gain. The horn length, however, can be considerably reduced by using a phase correcting lens that cancels the phase error introduced by the increase in flare angle, mandatory to reduce horn length.

In this paper, the design, simulation and measurement of a large $\mathrm{C}$ band horn, to be used in a radio telescope for VLBI (Very Large Base Interferometry) applications as well as for remote sensing calibration, are exposed. Given the frequency narrow band nature of these uses, the proposed antenna is a dual mode Potter horn combined with a septum polarizer to obtain the circular polarization. Specifically, the main specifications of the horn are listed:
- Low SLL (-30 dB) and high beam efficiency.

- $22^{\circ}$ beamwidth at $-10 \mathrm{~dB}$.

- LHCP and RHCP input ports with axial ratio lower than $0.2 \mathrm{~dB}$.

- Return loss and port isolation below $-20 \mathrm{~dB}$.

- C-band: 5.2-5.4 GHz.

The paper is organized as follows. In section II, a discussion of two different types of lenses and their effects on the radiation pattern is given so as to choose the best one. In section III, the design and simulation of the horn and its polarizer is summarized, and in section IV measurement results are given and discussed. Finally, in section $\mathrm{V}$ some conclusions are drawn.

\section{LENS THEORETICAL ANALYSIS}

One-surface lenses (meniscus and plane-convex, see Fig.1) are used to correct phase error of large horns to reduce their length. The basic design of theses lenses is discussed extensively in literature [2]. Particularly, lenses used with corrugated horns are discussed in [3], [4]. For our design, a narrow band application, a Potter horn [5] has been used. In order to select the lens better fits our application, a MATLAB code based on the classical expressions of the aperture fields and geometrical optics has been developed to derive the lens horn theoretical radiation patterns. The developed code results have been contrasted with the ones presented in [3], [4] showing good agreement.

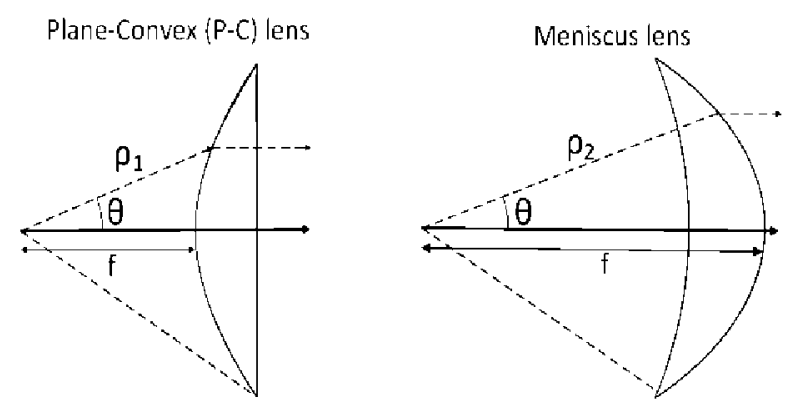

Fig. 1. Types of lenses considered

In the lens horn design, the first step is to calculate the approximate aperture diameter needed to obtain the desired beamwidth or gain $\left(-10 \mathrm{~dB}\right.$ at $22^{\circ}$, i.e. $\left.23 \mathrm{dBi}\right)$. In this particular case this value is around $30 \mathrm{~cm}$. A Potter horn 
without lens, with a phase error of 0.15 , results in an impractical length of $1.5 \mathrm{~m}$. As mentioned before, this length can be reduced significantly, with the inevitable effect of increasing the aperture phase error (in our case up to $\mathrm{s}=0.5$, with a horn length of around $40 \mathrm{~cm}$ ). This shorting distorts completely the radiation pattern, as can be seen in Fig. 2. Using dielectric lenses to transform the spherical wave front incoming from the horn vertex into a planar front, equiphasic aperture distributions are obtained, and the radiation patterns are focused.

Table 1 lists the calculated lengths and aperture diameters required to meet the $22^{\circ}$ beamwidth at $-10 \mathrm{~dB}$ for the three horns considered: a large horn without lens, a meniscus lens horn and a plane-convex lens horn. As it can be seen, the use of lenses renders horns around four times shorter. The differences in length and diameter are due to the different amplitude aperture distributions in the lens horns.

For better understanding of the different effects of both lenses, the aperture fields of each horn have been computed and plotted in Fig. 3. Plane-convex lenses tend to concentrate the rays in the centre whereas the meniscus ones have the opposite effect, obtaining a more uniform illumination. The field of the bare horn corresponds to the Potter propagating modes with no alteration.

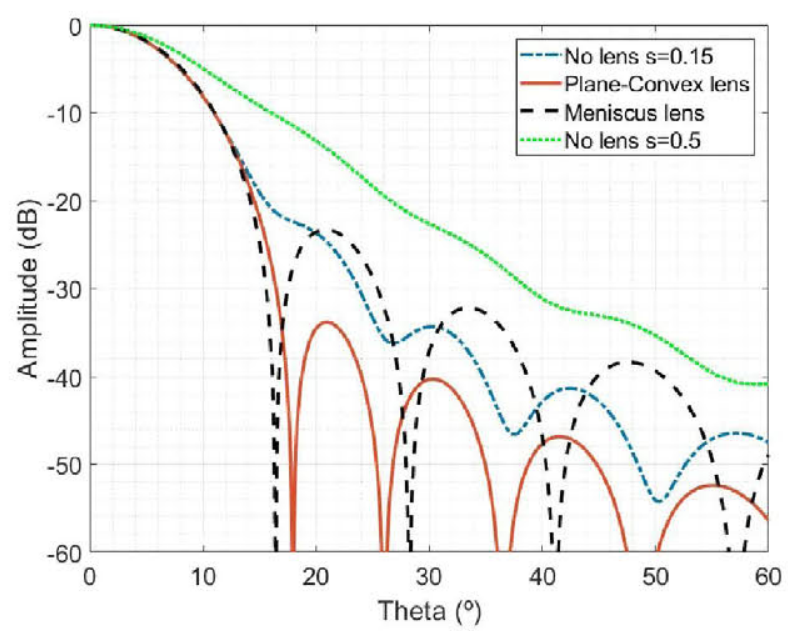

Fig. 2. Lens comparative between radiation patterns of the two type of lenses, a horn with low phase error $(0.15)$ and with high phase error $(0.5)$.

Table 1. Dimensions and spill-over outside $22^{\circ}$ beamwidth for the different horns

\begin{tabular}{|l|l|l|l|}
\hline \multirow{2}{*}{} & \multicolumn{3}{|c|}{ Horn type } \\
\cline { 2 - 4 } & Bare horn & $\begin{array}{l}\text { Meniscus } \\
\text { lens horn }\end{array}$ & $\begin{array}{l}\text { Plane convex lens } \\
\text { horn }\end{array}$ \\
\hline $\begin{array}{l}\text { Diameter } \\
(\mathrm{cm})\end{array}$ & 35 & 32 & 36 \\
\hline $\begin{array}{l}\text { Length } \\
(\mathrm{cm})\end{array}$ & 150 & 39 & 43 \\
\hline $\begin{array}{l}\text { Spill-Over } \\
(\mathrm{dB})\end{array}$ & 0.5 & 0.42 & 0.3 \\
\hline
\end{tabular}

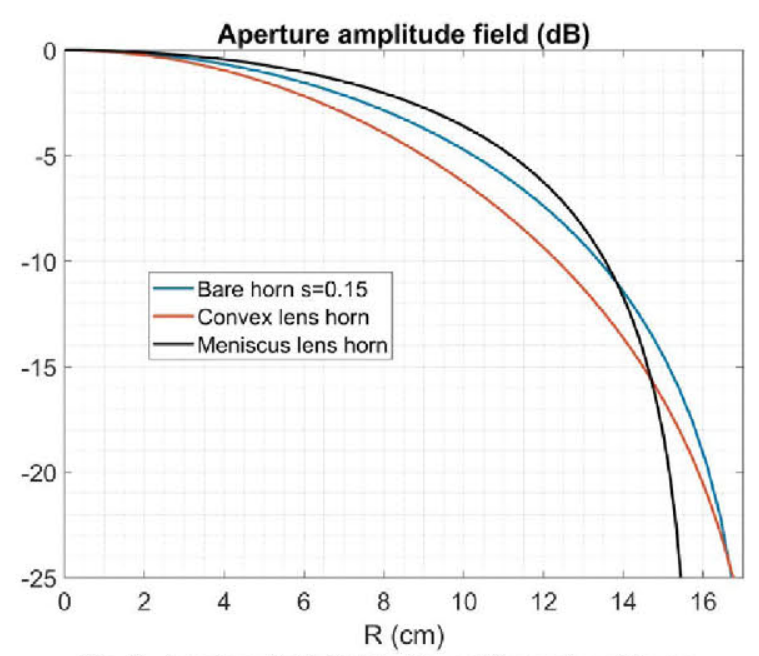

Fig. 3. Aperture field distribution of the analysed homs

The aperture field conditions the horn radiation. The computed radiation patterns are plotted in Fig. 2, showing important differences in the SLL. The more tapered distribution of the plane-convex lens produces a low SLL while the meniscus exhibits a higher level, as it is closer to a uniform illumination. The bare horn shows an intermediate behaviour and the phase error can be appreciated since the radiation pattern lacks nulls.

Table 1 also lists the spill-over losses outside the $22^{\circ}$ beamwidth for each horn. As it can be seen, the plane-convex lens horn is the most appropriate to obtain a compact size with the lowest SLL and spill-over. The meniscus obtains the same beamwidth with $10 \%$ less of diameter but increases the SLL. This SLL improvement is only obtained for small flare angles $\left(15^{\circ}-20^{\circ}\right)$ and it reduces as the angle increases due to the fact that the lens concentrates too much the power in the aperture centre widening excessively the main lobe, as explained in [3].

\section{ANTENNA DESIGN: HORN AND POLARIZER}

A sectional view of the designed antenna is shown in Fig. 4. It consists of the plane-convex lens loaded horn and a septum polarizer to obtain the circular polarization. In this section the main design steps are outlined.

\section{A. Lens Horn Design}

Having chosen the plane convex lens horn, its design comprises the following steps:

\section{1) Horn Design and Optimization}

The design of Potter horns has been analysed extensively in literature [5], [6]. The horn aperture diameter is derived from the desired beamwidth $(36 \mathrm{~cm})$ and the $\mathrm{TE}_{11}+\mathrm{TM}_{11}$ mode converter section is designed and optimized to obtain appropriate matching and an acceptable level of cross polarization. The horn length can be considerably reduced as the phase error will be corrected by the lens, but as previously mentioned, high flare angles tend to perform badly in terms of SLL. Therefore $43 \mathrm{~cm}\left(19^{\circ}\right)$ have been chosen as a compromise. 


\section{2) Lens Design}

Geometrical optics formulation is used to derive the expression of the lens surface [2] to correct the phase error of the horn. To implement the plane-convex lens, Teflon $\left(\varepsilon_{\mathrm{r}}=2.1\right)$ has been selected due to its good properties in terms of water absorption (hydrophobic material), UV radiation resilience and low dissipation factor (0.0002 loss tangent) at C-band frequencies.

\section{3) Lens Matching}

The lens creates a dielectric discontinuity resulting in a high reflection coefficient in both lens faces. Therefore, matching techniques have to be applied. This is achieved by quarter wavelength impedance transformer layers placed over the front and rear lens surfaces. These transformers must have a dielectric constant equal to the geometric mean of the air and Teflon permittivities $\left(\sqrt{\epsilon_{r}}\right)$. The transformer layers are implemented using Teflon too, but applying cylindrical holes to it, to reach the desired effective dielectric constant [7]. This results in the matched lens depicted in Fig. 4, where the perforations can be seen. A good optimization of these matching layers is very important to reach the low axial ratio specification, as it will be explained later.

\section{B. Polarizer Design and Optimization}

To obtain circular polarization, a square waveguide septum polarizer is used, as it acts as orthomode transducer and polarizer at the same time. The polarizer has two SMA coaxial inputs as RHC and LHC ports. The design of this type of polarizers is dealt in the literature [8], [9]. To connect the polarizer to the horn, a single step rectangular to circular waveguide transition is used. A detail of the septum geometry is depicted in Fig 4.

In order to assess the mechanical precision required to meet the axial ratio specification, a tolerance test has been carried out concluding that a value below $50 \mu \mathrm{m}$ is needed. However, to reach the $0.2 \mathrm{~dB}$ of axial ratio in the complete antenna, not only the septum parameters have to be taken into account, but also the lens horn adaptation, as it will be explained in section C.
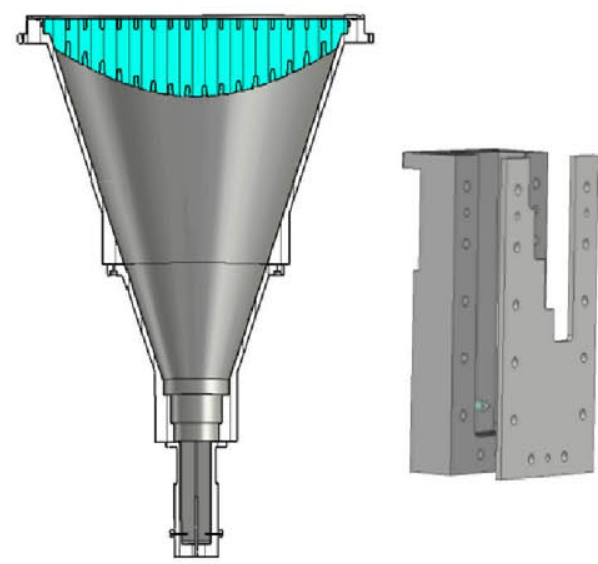

Fig. 4. Sectional view of the designed horn and explosion of septum polarizer

\section{Polarizer and Horn Assembly}

Once the septum and Potter horn have been designed, the last step is their assembly. At this moment, the overall return loss and port isolation must be optimized to maintain the low axial ratio of the septum itself. This is a typical issue when working with circular polarizations: the axial ratio is degraded if the overall RHC/LHC port isolation is poor or these are not well matched. This is because the power coupled in the opposite port, if it is not completely absorbed, is reradiated as cross polar polarization.

The isolation can be improved by adjusting the length of the cylindrical waveguide section connecting the rectangular to circular transformer and the horn. As a circular polarization changes its rotation direction when reflected, the horn return loss contributes to the total port isolation. This means that the complete system return loss will be roughly the same as that of the septum, while the isolation will be a combination of the septum isolation and horn reflection coefficient. So, choosing carefully the length of the intermediate waveguide section, this combination can be made destructive maximizing the port isolation. As previously mention, this step is crucial to maintain a good axial ratio, resulting in simulated values below $0.1 \mathrm{~dB}$.

\section{FABRICATION AND MEASUREMENT}

The septum polarizer, lens and horn have been manufactured and assembled for measurement (see Fig. 5). Return loss and port isolation are shown in Fig. 6 where it can be seen that they are below $-20 \mathrm{~dB}$ by far in the operating bandwidth. However, there exists a displacement in the resonance between the measured return loss of the two ports and with the simulation. These displacements are due to the very high sensibility of the resonance associated to the coaxial to waveguide transition.

Radiation patterns have been measured in anechoic chamber showing excellent agreement with the simulation as seen in Fig. 7. In particular the side lobe level is below $31 \mathrm{~dB}$ and the far radiation is extremely low, which is of great importance in order to mitigate multipath signals and spillover. The measured gain is $23.4 \mathrm{dBi}$ and dielectric losses are estimated to be below $0.1 \mathrm{~dB}$.

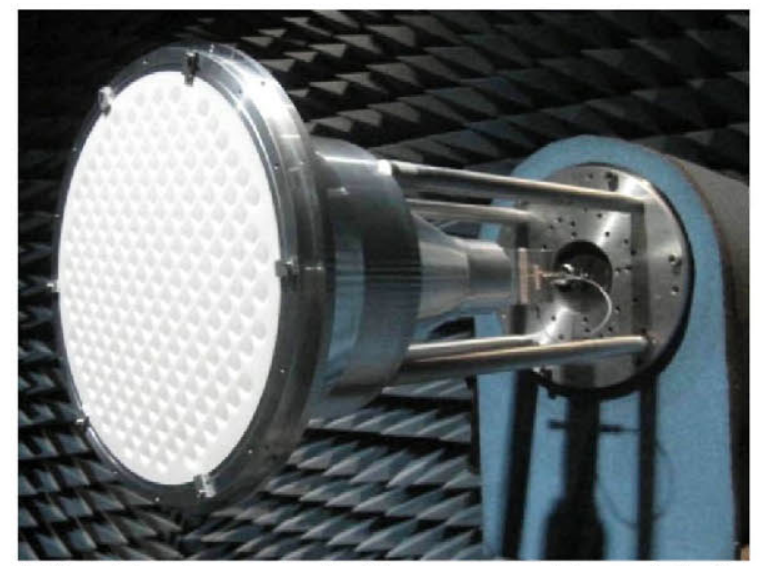

Fig. 5. Complete antenna assembled for measurement in anechoic chamber 
Axial ratio at boresight is around $0.1 \mathrm{~dB}$ which constitutes an extraordinary low level, as can be seen in Fig. 8, where the worst cases obtained during the tolerance test have been superposed. To understand better the exceptionally good results, some measurement error sources must be considered, such as the positioner rotary joint insertion loss variation of the measurement setup ( $0.05 \mathrm{~dB}$ typically) and the imperfect load in the cross polar port.

\section{CONCLUSION}

A narrow beam lens horn antenna suitable for remote sensing calibration and Cassegrain feeding has been designed, simulated and measured. The main design challenge is the lens design, as its shape conditions the side lobe level and antenna adaptation. An analytical study of the lenses effects has been carried to select the plane convex lens as the type that better fits our application. To operate with circular polarization, a septum polarizer has been designed and mechanized, showing an axial ratio of only $0.1 \mathrm{~dB}$. Radiation pattern and scattering parameters have been measured as well, showing good agreement with the simulations and meeting the required specifications.

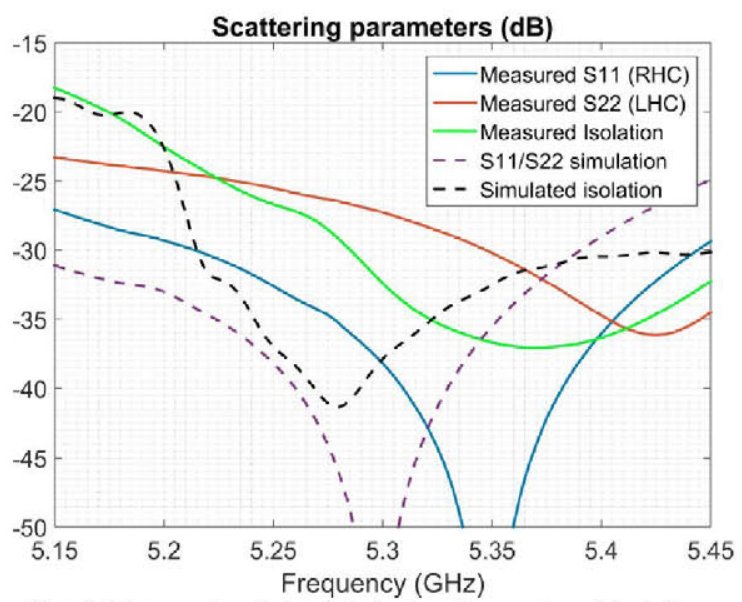

Fig. 6. Measured and simulated return loss and port isolation

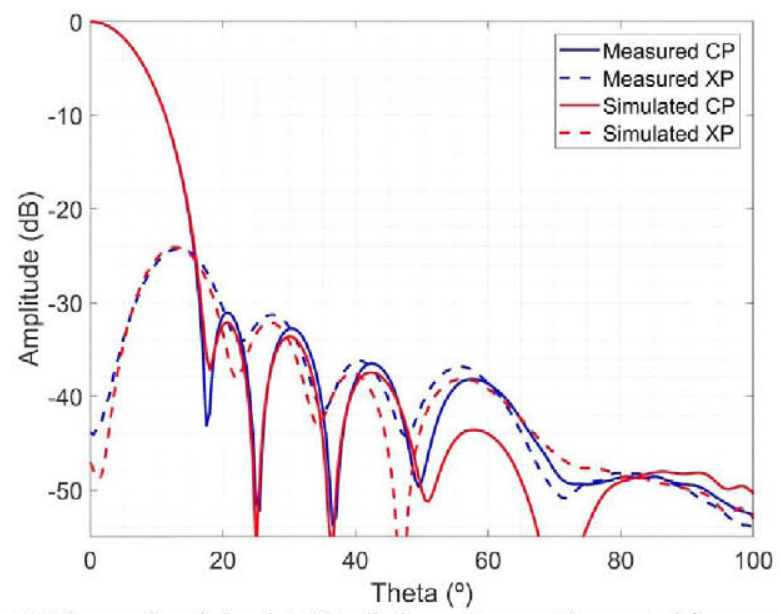

Fig. 7. Measured and simulated Radiation patterns at the central frequency band $(5.3 \mathrm{GHz})$

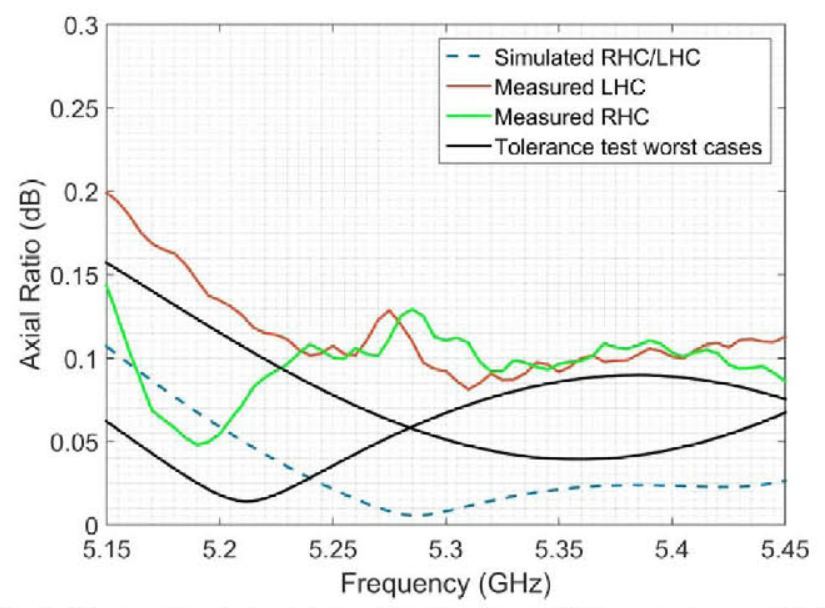

Fig. 8. Measured and simulated axial ratio along with the worst cases obtained during the $50 \mu \mathrm{m}$ tolerance test

\section{ACKNOWLEDGMENT}

This work has been supported by the Technical University of Madrid (UPM). In addition, the authors would like to acknowledge the Spanish Government, Ministry of Economy, National Program of Research, Development and Innovation for the support of this publication in the projects ENABLING5G "ENABLING INNOVATIVE RADIO TECHNOLOGIES FOR 5G NETWORKS" (project number TEC2014-55735-C31-R) and FUTURE-RADIO "Radio systems and technologies for high capacity terrestrial and satellite communications in an hyperconnected world" (project number TEC2017-85529-C3$1-\mathrm{R})$.

\section{REFERENCES}

[1] Ad Stoffelen, "A Simple Method for Calibration of a Scatterometer over the Ocean", Journal of Atmospheric and Oceanic Technology, $16(2)$.

[2] Thomas A. Milligan, "Modern Antenna Design", Second Edition, 447451.

[3] Clarricoats, P.J.B., and Saha, P.K.: 'Radiation patterns of a lenscorrected conical scalar horn', Electron. Lett., 1969, 5, pp. 592-593.

[4] P-S. Kildal, K. Jakobsen, K. S. Rao, "Meniscus lens-corrected corrugated horn: An efficient feed for a Cassegrain antenna", Proc. Inst. Elec. Eng., no. 6, pp. 390-394, 1984-Dec.

[5] P.D. Potter ."A new horn antenna with suppressed sidelobes and equal beamwidths". Microwave J., Vol VI, No. 6, pp. 71 - 78, June 1963.

[6] A. D. Olver, P. J. B. Clarricoats, A. A. Kishk and L Shafai, "Microwave Horns and Feeds", IEE Electromagnetic Wave Series, New York, USA, 1994.

[7] MORITA, T., and COHN, S.B.: 'Microwave lens matching by simulated quarterwave transformers', IRE Trans., 1956, AP-4, pp. 3339.

[8] M. H. Chen, G. N. Tsandoulas, "A wide-band square-waveguide array polarizer", IEEE Trans. Antennas Propagat., vol. AP-21, pp. 389-391, May 1973.

[9] Zhong, W., B. Li, Q. Fan, and Z. Shen, "X-band compact septum polarizer design," ICMTCE, 167-170, 2011. 